\title{
Risk-Benefit Analysis of Pediatric-Inspired Versus Hyperfractionated Cyclophosphamide, Vincristine, Doxorubicin, and Dexamethasone Protocols for Acute Lymphoblastic Leukemia in Adolescents and Young Adults
}

\author{
Gregory F. Guzauskas, MSPH, PhD,' Kathleen F. Villa, MS, Geertrui F. Vanhove, MD, PhD, \\ Vicki L. Fisher, and David L. Veenstra, PharmD, PhD ${ }^{1}$
}

\begin{abstract}
Purpose: To estimate the risk-benefit trade-off of a pediatric-inspired regimen versus hyperfractionated cyclophosphamide, vincristine, doxorubicin, and dexamethasone (hyper-CVAD) for first-line treatment of adolescents/young adult (AYA; ages 16-39 years) patients with Philadelphia-negative acute lymphoblastic leukemia. Methods: Patient outcomes were simulated using a 6-state Markov model, including complete response (CR), no $\mathrm{CR}$, first relapse, second CR, second relapse, and death. A Weibull distribution was fit to the progression-free survival curve of hyper-CVAD-treated AYA patients from a single-center study, and comparable patient data from a retrospective study of pediatric regimen-treated AYA patients were utilized to estimate a relative progression difference (hazard ratio $=0.51$ ) and model survival differences. Health-state utilities were estimated based on treatment stage, with an assumption that the pediatric protocol had 0.10 disutility compared with hyper-CVAD before the maintenance phase of treatment. Total life-years and quality-adjusted life-years (QALYs) were compared between treatment protocols at 1,5 , and 10 years, with additional probabilistic sensitivity analyses.

Results: Treatment with the pediatric-inspired protocol was associated with a 0.04 increase in life-years, but a 0.01 decrease in QALYs at 1 year. By years 5 and 10, the pediatric-inspired protocol resulted in 0.18 and 0.24 increase in life-years and 0.25 and 0.32 increase in QALYs, respectively, relative to hyper-CVAD. The lower quality of life associated with the induction and intensification phases of pediatric treatment was offset by more favorable progression-free survival and overall survival relative to hyper-CVAD.

Conclusions: Our exploratory analysis suggests that, compared with hyper-CVAD, pediatric-inspired protocols may increase life-years throughout treatment stages and QALYs in the long term.
\end{abstract}

Keywords: acute lymphoblastic leukemia, hyper-CVAD, Markov model, pediatric inspired, progression-free survival, risk-benefit

\section{Introduction}

A CUTE LYMPHOBLASTIC LEUKEMIA (ALL), a serious and potentially fatal hematologic disorder, is the most common malignancy in the pediatric population, accounting for almost $30 \%$ of childhood cancers and $6 \%$ of cancers in adolescents. ${ }^{1}$ While the highest incidence of ALL is among children aged $<5$ years, ${ }^{2}$ treatment outcomes in adolescents and young adults (AYAs, ages 16-39 years) are poorer compared with childhood outcomes due to unfavorable cytogenetic features associated with increasing age, a higher incidence of precursor T-cell immunophenotype, higher hemoglobin levels at diagnosis, and a lower incidence of lymphomatous features in AYAs. ${ }^{3-5}$

Among the prognostic cytogenetic features of ALL is the presence of the Philadelphia chromosome, which is strongly associated with poorer outcomes. ${ }^{6}$ Most of the available clinical evidence for treatment and outcomes in the AYA

\footnotetext{
${ }^{1}$ Pharmaceutical Outcomes Research and Policy Program, Department of Pharmacy, University of Washington, Seattle, Washington.

${ }^{2}$ Jazz Pharmaceuticals, Palo Alto, California.

Portions of the data in this article were presented as a poster at the 2014 American Society of Hematology in San Francisco, CA; December 6-9, 2014. Poster 1281; abstract published in Blood 2014;124(21):Abstract 1281.

(C) Gregory F. Guzauskas et al., 2017; Published by Mary Ann Liebert, Inc. This Open Access article is distributed under the terms of the Creative Commons Attribution Noncommercial License (http://creativecommons.org/licenses/by-nc/4.0/), which permits any noncommercial use, distribution, and reproduction in any medium, provided the original author(s) and the source are credited.
} 
population is for Philadelphia-negative patients since only $\sim 5 \%-25 \%$ of AYA patients have Philadelphia-positive ALL, ${ }^{7}$ although this frequency is notably higher compared with pediatric ALL patients $(2 \%-3 \%) .{ }^{8,9}$ While recent improvements in early detection and treatment of ALL have led to substantial survival gains in the overall ALL population in the United States, survival remains relatively poor in AYA patients, ${ }^{10}$ likely due, at least in part, to differences in treatment approaches and oncology team experience. ${ }^{5}$

Hyperfractionated cyclophosphamide, vincristine, doxorubicin, and dexamethasone (hyper-CVAD), which has demonstrated efficacy in ALL and in other hematologic malignancies, including Burkitt's disease, lymphoblastic lymphoma, mantle cell lymphoma, and multiple myeloma, is a commonly used protocol in the adult ALL population. ${ }^{11,12}$ Alternatively, pediatric regimens have shown improved outcomes in AYA patients treated with intensified postremission strategies in adult cooperative group studies, however, these regimens have not been directly compared with hyper-CVAD regimens. ${ }^{13-16}$ Pediatric-inspired protocols utilize higher doses of nonmyelosuppressive agents, including L-asparaginase, a drug that has been used in pediatric ALL therapy since the 1960s. ${ }^{13,15}$ Nonetheless, pediatric protocols are thought to be less tolerable and to lead to increased adverse events in AYAs, relative to pediatric patients. ${ }^{16,17}$

Additional issues with determining pediatric-protocol outcomes in AYAs include the following: (1) ALL is relatively uncommon in this population, meaning these patients are often treated in clinical practice by either pediatric or adult oncologists who may use different protocols, and (2) AYAs have low rates of access to and accrual on clinical trials. ${ }^{18}$ Thus, not only are pediatric protocols less likely to be used in AYAs in clinical practice but to date, no randomized controlled trials have comparatively studied a pediatric regimen versus an adult regimen such as hyper-CVAD in the AYA population.

A quantitative evaluation of the risks and benefits of pediatric versus hyper-CVAD therapy in AYA ALL patients may provide useful guidance for patient-specific treatment decisions and development of treatment guidelines. The objective of this analysis was to estimate the risk-benefit trade-off of a pediatric-inspired regimen compared with hyper-CVAD for first-line treatment of AYA patients with Philadelphia-negative ALL. We thus used a decisionanalytic model to ascertain the relative risks and benefits of these strategies to inform clinicians and other decision makers in the absence of a definitive clinical trial.

\section{Methods}

\section{Model rationale}

Our initial aim was to model clinical-trial evidence from a direct comparison of pediatric regimens with hyper-CVAD in the target population. We first performed a literature search to identify clinical trials evaluating the use of hyper-CVAD in the treatment of AYA patients with ALL. We searched PubMed using the keywords "hyperCVAD" or "hyper-CVAD" and "acute lymphoblastic/lymphocytic leukemia/lymphoma." Abstracts from all English language clinical trials published between 2000 and 2013 were reviewed and manually evaluated for relevance. A total of 23 relevant publications were identified; however, no prospective comparison of pediatric- inspired versus hyper-CVAD treatment protocols was found. We thus focused on identifying studies with comparable firstline Philadelphia-negative AYA ALL patient populations from the two treatment protocols of interest. Because no clinical trials directly comparing pediatric-inspired versus hyper-CVAD outcomes were identified, we chose a decisionanalytic-modeling framework that utilized patient data from two population-appropriate studies ${ }^{19,20}$ and made simplifying assumptions to structure our exploratory analysis.

Decision-analytic modeling provides a structured method for quantitatively assessing complex clinical data that is also useful for gaining a better understanding of the impact of data uncertainty. ${ }^{21}$ Such a model enables indirect comparisons of outcomes under different treatment conditions and can be used for evaluation of life expectancy and quality-adjusted life expectancy. Furthermore, incremental benefit can be estimated using the metric of quality-adjusted life-years (QALYs), which provides a pragmatic way to combine two dimensions of health-life duration and health status-into an index that allows for direct comparison of benefits and harms. The incremental health benefit of a treatment in comparison with standard treatments is calculated as the incremental difference between effectiveness and risk (both measured using QALYs) at specified time points. A favorable risk-benefit balance exists when the expected QALY gains as a result of treatment efficacy exceed the expected losses from treatment risks (e.g., adverse events) in order for the net health benefit to be positive. ${ }^{21}$

\section{Model structure}

This analysis used a 6-state Markov model (Fig. 1) to evaluate the risks and benefits of using a pediatric-inspired protocol compared with hyper-CVAD. In the model, patients could transition every month among the potential outcomes of initial complete response, no complete response, first relapse, second complete response, second relapse, and death. Following induction therapy, hypothetical patients entered the

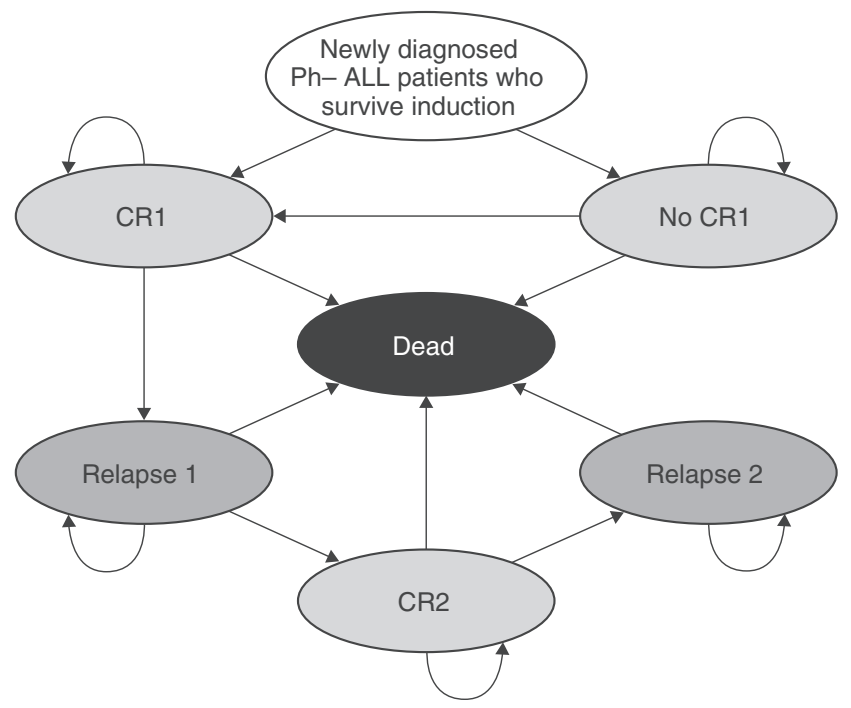

FIG. 1. Markov health-state transition model. Patients could transition among the health states at intervals of 1 month, with a total time horizon of 10 years. ALL, acute lymphoblastic leukemia; CR1, initial complete response; CR2, second complete response; $\mathrm{Ph}-$, Philadelphia-negative. 


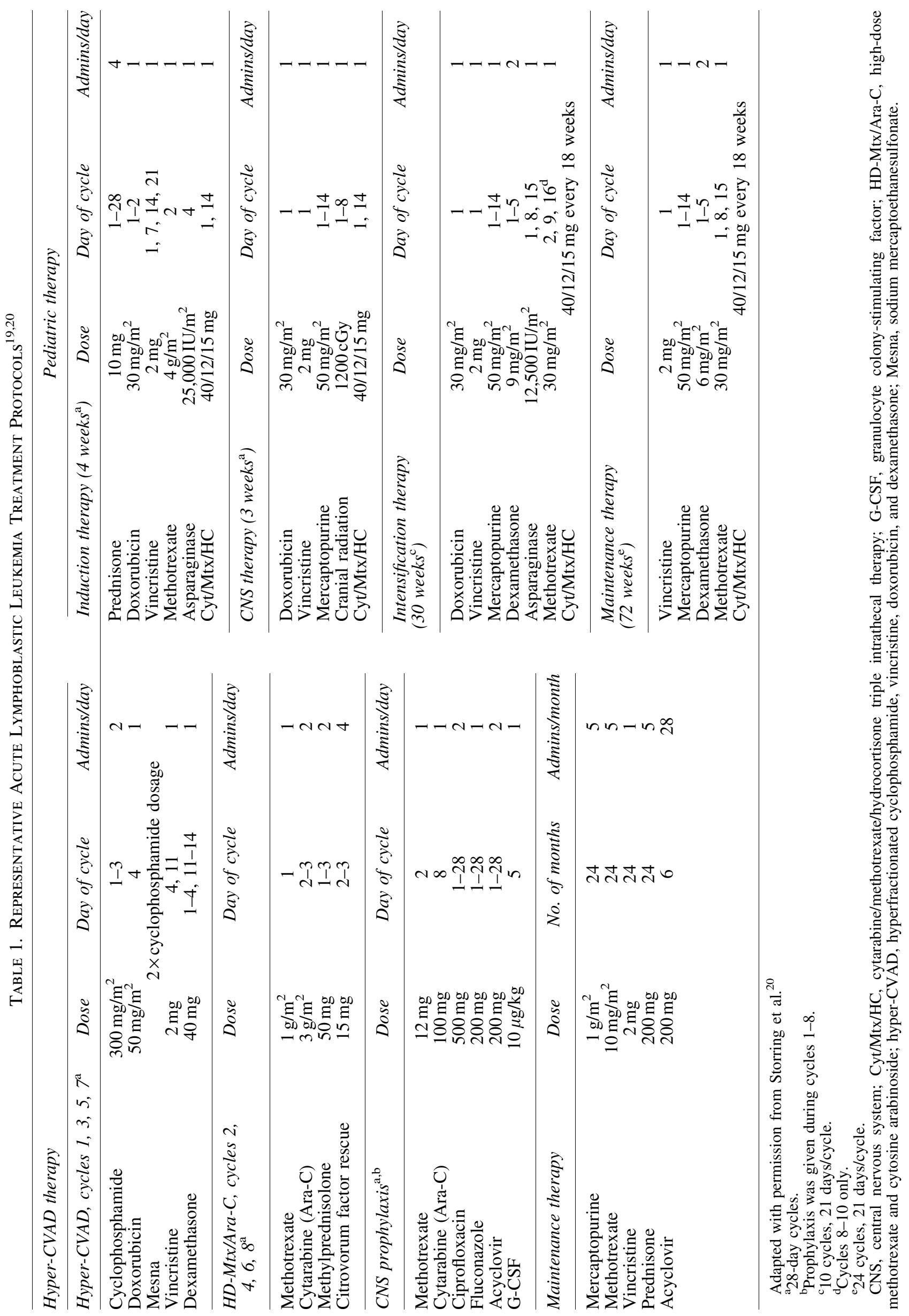




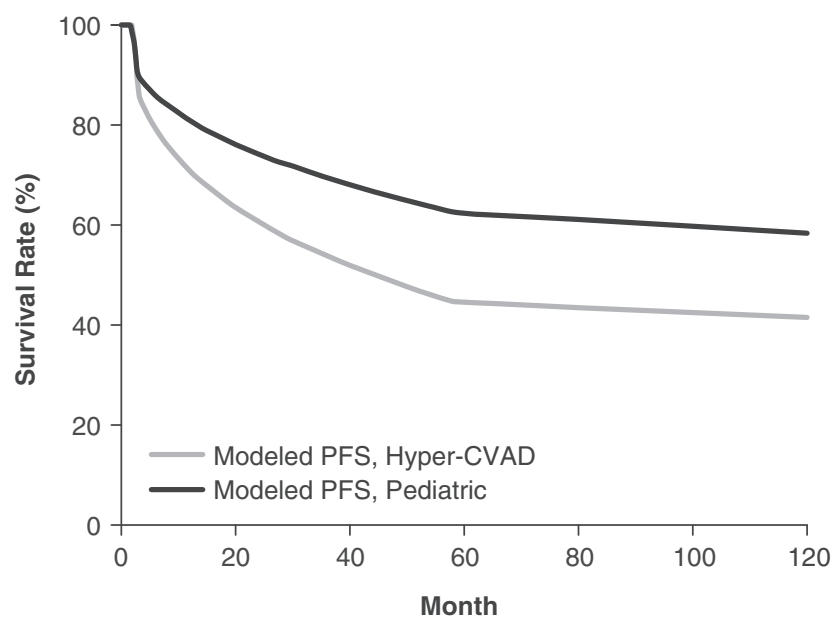

FIG. 2. Estimation of progression-free survival for model input based on studies of hyper-CVAD ${ }^{19}$ or pediatric-inspired protocols. ${ }^{20}$ Hyper-CVAD, hyperfractionated cyclophosphamide, vincristine, doxorubicin, and dexamethasone; PFS, progression-free survival.

model in either the complete response or no complete response health state based on response data from the two AYA studies $^{19,20}$; patients could remain in their current health state, experience a remission, experience a relapse, or die. We limited our analysis to a 10-year time horizon, since curve fits may overestimate progression-free survival changes beyond study-reported follow-up time, with an assumption that the risk of relapse ended if a patient spent 5 years in the initial complete response health state. Model evaluations were performed for the cumulative time periods of 1,5 , and 10 years.

\section{Population}

Patients modeled for the hyper-CVAD protocol (Table 1) were from a single-center, long-term, follow-up study of hyper-CVAD in 288 patients, of whom $17 \%$ were Philadelphia chromosome-positive. ${ }^{19}$ While the median age of the patients in the overall study was 40 years, the 5-year survival outcomes (although not survival curves) were stratified by age, enabling data extraction for the population aged $<40$ years; the 5-year progression-free survival rate for patients aged $<40$ years was $44 \%$. In the absence of further stratification of this age group by Philadelphia-chromosome status, we made the simplifying assumption that this survival rate was equivalent to that of a Philadelphia-negative population.

For the pediatric protocol, comparable patient data were used from a retrospective study of 85 patients treated with a pediatricinspired regimen (Table 1). ${ }^{20}$ Patients in this study had a median age of 37 years (18-60 years), all were Philadelphia chromosome-negative, and were initiated on a modified Dana Farber Cancer Institute pediatric protocol (DFCI 91-01). Age-stratified data for the population aged $<35$ years were used for the model to reflect the AYA population. The 3-year progression-free survival rate was $77 \%$ for patients aged $\leq 35$ years and $60 \%$ for patients aged $>35$ years. Because the reference hyperCVAD population was aged $<40$ years, we assumed patients aged $<40$ years on the pediatric protocol had a 3-year progression-free survival of $70 \%$, which is between the stratified estimates for patients aged $\leq 35$ and $>35$ years.

Table 2. Parameters Used in the Base Case and Probabilistic Sensitivity Analyses

\begin{tabular}{|c|c|c|c|}
\hline \multirow[b]{2}{*}{ Parameter } & \multirow[b]{2}{*}{$\begin{array}{c}\text { Base case, } \\
\text { value (range) }\end{array}$} & \multicolumn{2}{|c|}{ Probabilistic sensitivity analysis } \\
\hline & & $\begin{array}{l}\text { Distribution } \\
\text { type }\end{array}$ & Source \\
\hline \multicolumn{4}{|l|}{ Hyper-CVAD outcomes } \\
\hline Probability induction death & $0.02(0.00-0.04)$ & Beta & Kantarjian et al. ${ }^{19}$ \\
\hline Probability complete response & $0.95(0.92-0.99)$ & Beta & Kantarjian et al. ${ }^{19}$ \\
\hline Probability complete response after 1 course & $0.81(0.85-0.87)$ & Beta & Kantarjian et al. ${ }^{19}$ \\
\hline 5 -year progression-free survival & $0.44(0.36-0.52)$ & Beta & Kantarjian et al. ${ }^{19}$ \\
\hline \multicolumn{4}{|l|}{ Pediatric outcomes versus hyper-CVAD } \\
\hline Relative risk induction death & $1.50(0.15-15.22)$ & Log-normal & $\begin{array}{l}\text { Kantarjian et al. }{ }^{19} \\
\text { and Storring et al. }\end{array}$ \\
\hline Relative risk complete response & $1.03(0.97-1.09)$ & Log-normal & $\begin{array}{l}\text { Kantarjian et al. }{ }^{19} \\
\text { and Storring et al. }\end{array}$ \\
\hline Hazard ratio progression-free survival & $0.51(0.41-0.61)$ & Log-normal & Assumption \\
\hline \multicolumn{4}{|l|}{ Global outcomes (both therapies) } \\
\hline Month progression risk ends & $60(48-72)$ & Normal & Assumption \\
\hline Probability of progression survival & $0.93(0.86-0.99)$ & Beta & Storring et al. ${ }^{20}$ \\
\hline Probability second complete response & $0.09(0.00-0.26)$ & Beta & Storring et al. ${ }^{20}$ \\
\hline Probability second progression & $0.90(0.83-0.97)$ & Beta & Assumption \\
\hline Percent transplant candidates pediatric & $0.16(0.13-0.19)$ & Beta & Storring et al. ${ }^{20}$ \\
\hline Percent transplant candidates hyper-CVAD & $0.42(0.33-0.50)$ & Beta & Kantarjian et al. ${ }^{19}$ \\
\hline \multicolumn{4}{|l|}{ Utility parameters } \\
\hline Induction & $0.65(0.52-0.78)$ & Beta & Assumption \\
\hline Intensification & $0.60(0.48-0.72)$ & Beta & Assumption \\
\hline Maintenance & $0.75(0.60-0.90)$ & Beta & Assumption \\
\hline Posttherapy & $0.85(0.68-1.00)$ & Beta & Assumption \\
\hline Progression & $0.60(0.48-0.72)$ & Beta & Assumption \\
\hline
\end{tabular}


Table 3. Model Results of Life-Years and Quality-Adjusted Life-Years Comparisons

\begin{tabular}{lccc}
\hline & \multicolumn{3}{c}{ Value 95\% credible range $)^{\mathrm{a}}$} \\
\cline { 2 - 4 } Outcome & Pediatric-inspired protocol & Hyper-CVAD protocol & Difference $^{\mathrm{b}, \mathrm{c}}$ \\
\hline $\begin{array}{l}1 \text { year } \\
\text { Life-years }\end{array}$ & $0.82(0.80-0.83)$ & $0.77(0.75-0.79)$ & $0.04(0.03-0.06)$ \\
$\quad$ QALYs & $0.51(0.43-0.58)$ & $0.51(0.43-0.59)$ & $-0.01(-0.09$ to 0.08$)$ \\
5ears & & & \\
$\quad$ Life-years & $1.88(1.73-2.01)$ & $1.63(1.45-1.79)$ & $0.25(0.15-0.39)$ \\
$\quad$ QALYs & $1.36(1.16-1.55)$ & $1.18(0.97-1.37)$ & $0.18(0.05-0.33)$ \\
10 years & & $1.80(1.57-2.01)$ & $0.32(0.18-0.49)$ \\
$\quad$ Life-years & $2.12(1.92-2.29)$ & $1.32(1.09-1.55)$ & $0.24(0.09-0.42)$ \\
$\quad$ QALYs & $1.56(1.32-1.80)$ & & \\
\hline
\end{tabular}

a $95 \%$ credible range derived from probabilistic sensitivity analysis.

${ }^{\mathrm{b}}$ Calculated as pediatric protocol minus hyper-CVAD protocol.

${ }^{c}$ Values rounded to the nearest 100th.

QALYs, quality-adjusted life-years.

\section{Model assumptions and inputs}

The two primary assumptions we used in developing the model were that (1) results are driven by progression-free survival such that an overall survival advantage is due to a difference in relapse, and (2) after the first relapse, survival trajectory would be equal for both protocols. Hyper-CVAD progression-free survival was modeled by fitting a Weibull distribution to the progression-free survival curve of the overall hyper-CVAD population. We then adjusted the hyper-CVAD curve so that the 5-year progression-free survival matched the age-stratified estimate of $44 \%$. Based on the progression-free survival point estimates in the two groups, we calculated a hazard ratio of 0.51 , fitting a second curve for the pediatric-inspired treatment group to reflect that hazard ratio between the two groups. We modeled a wide uncertainty range in the hazard ratio $( \pm 40 \%)$ due to the assumptive nature of the survival estimates (Fig. 2).

We were unable to identify utility estimates specific to ALL in the AYA population through review of the published literature. Health-state utilities were thus estimated based on comparison with other leukemia studies and differentiated by (1) treatment stage, and (2) progression-free versus progressed states, with the assumption that the pediatric-inspired protocol had a 0.10 disutility in progression-free survival compared with hyper-CVAD before the maintenance phase of treatment. This disutility was incorporated because of the perceived potential for increased adverse events and intolerability. We assumed that adverse event-specific disutilities associated with treatment were implicit in this estimate. The utilities were then applied to treatment stage and overall survival to calculate overall QALYs for both treatment protocols.

\section{Analysis}

The primary outcomes were total life-years and total QALYs for each regimen, estimated in the base case using values derived from the two observational AYA ALL studies as described above (Table 2). These estimated values were then used to determine the incremental health benefits for the pediatric-inspired protocol relative to hyper-CVAD, defined as the estimated life-years and QALYs from the pediatric protocol minus the life-years and QALYs from the hyper-
CVAD protocol. In addition, we conducted one-way (univariate) and probabilistic sensitivity analyses to evaluate the uncertainty associated with all model parameters. In the probabilistic sensitivity analysis, parameters were assigned distributions with values as shown in Table 1 . The model was run for 10,000 simulations, and $95 \%$ credible ranges were calculated, that is, the interval for which the point estimate has a $95 \%$ probability of being contained.

\section{Results}

Within the first year, the pediatric-inspired protocol resulted in a gain of 0.82 life-years, while patients treated with hyper-CVAD gained 0.77 life-years, resulting in a difference of 0.04 (95\% credible range, 0.03-0.06) life-years favoring the pediatric-inspired protocol (Table 3). However, the pediatricinspired protocol was also associated with a slight decrease in QALYs gained within the first year, a decrease of -0.01 (95\% credible range, -0.01 to 0.01 ) QALYs relative to hyper-CVAD (Table 3 ).

By years 5 and 10, both the life-years gained and the QALYs gained with the pediatric-inspired protocol were greater than achieved with the hyper-CVAD protocol (Table 3). These greater gains resulted in incremental differences of $0.25(95 \%$ credible range, 0.15-0.39) life-years and 0.18 (95\% credible range, $0.05-0.33)$ QALYs at 5 years, and 0.32 (95\% credible range, 0.18-0.49) life-years and 0.24 (95\% credible range, $0.09-0.42$ ) QALYs at 10 years, favoring the pediatric-inspired protocol.

The model results were robust to parameter uncertainty. One-way sensitivity analysis at 10 years showed that the pediatric versus hyper-CVAD relative risk for complete response, the pediatric versus hyper-CVAD hazard ratio for progression-free survival, and utility estimates were the most influential parameters on incremental QALY results (Fig. 3A). For incremental life-years, the most influential parameters were likewise the pediatric versus hyper-CVAD relative risk for complete response and the pediatric versus hyper-CVAD hazard ratio for progression-free survival (Fig. 3B). None of the variability in individual parameters resulted in hyperCVAD having greater QALYs or life-years at 10 years. In probabilistic sensitivity analysis, the pediatric regimen had 


\begin{tabular}{|c|c|c|c|c|c|c|c|c|c|c|c|c|}
\hline \multicolumn{8}{|c|}{ A Incremental QALYs, 10-Year Model Timeframe } & \multirow[b]{2}{*}{$\begin{array}{l}\text { Low } \\
\text { Input }\end{array}$} & \multirow[b]{2}{*}{$\begin{array}{l}\text { High } \\
\text { Input }\end{array}$} & \multirow[b]{2}{*}{$\begin{array}{l}\text { Low } \\
\text { Result }\end{array}$} & \multirow[b]{2}{*}{$\begin{array}{c}\text { High } \\
\text { Result }\end{array}$} & \multirow[b]{2}{*}{ Spread } \\
\hline 0.16 & 0.19 & 0.22 & 0.25 & 0.28 & 0.31 & 0.34 & Parameter & & & & & \\
\hline & & & & & & & Pediatric RR CR & 0.97 & 1.09 & 0.17 & 0.34 & 0.17 \\
\hline & & & & & & & Pediatric HR PFS & 0.41 & 0.61 & 0.30 & 0.20 & 0.11 \\
\hline & & & & & & & Utility: PFS, Intensification & 0.48 & 0.72 & 0.19 & 0.30 & 0.11 \\
\hline & & & & & & & Utility: PFS, Post-Therapy & 0.68 & 1.00 & 0.19 & 0.30 & 0.11 \\
\hline & & & & & & & Utility: PFS, Induction & 0.52 & 0.78 & 0.30 & 0.20 & 0.10 \\
\hline & & & & & & & Pediatric RR Induction Death & 0.15 & 15.22 & 0.25 & 0.23 & 0.02 \\
\hline & & & & & & & Utility: PFS, Maintenance & 0.60 & 0.90 & 0.26 & 0.24 & 0.02 \\
\hline & & & & & & & Hyper-CVAD pr CR & 0.92 & 0.99 & 0.24 & 0.26 & 0.02 \\
\hline & & & & & & & Month Progression Risk Ends & 48 & 72 & 0.24 & 0.25 & 0.01 \\
\hline & & & & & & & $\%$ Transplant Candidates, Pediatric & 0.13 & 0.19 & 0.25 & 0.24 & 0.01 \\
\hline & & & & & & & $\%$ Transplant Candidates, Hyper-CVAD & 0.33 & 0.50 & 0.25 & 0.24 & 0.01 \\
\hline & & & & & & & pr 2nd CR & 0.00 & 0.26 & 0.25 & 0.25 & 0.00 \\
\hline & & & & & & & Hyper-CVAD pr Induction Death & 0.00 & 0.04 & 0.25 & 0.25 & 0.00 \\
\hline & & & & & & & pr 2nd Progression & 0.83 & 0.97 & 0.25 & 0.25 & 0.00 \\
\hline & & & & & & & pr Progression Survival & 0.86 & 0.99 & 0.25 & 0.25 & 0.00 \\
\hline & & & & & & & Utility: Progression & 0.48 & 0.72 & 0.25 & 0.25 & 0.00 \\
\hline & & & & & & & Hyper-CVAD pr CR After 1 Course & 0.75 & 0.87 & 0.25 & 0.25 & 0.00 \\
\hline & & & & & & & Hyper-CVAD 5-yr PFS & 0.36 & 0.52 & 0.25 & 0.25 & 0.00 \\
\hline \multicolumn{13}{|c|}{ B Incremental Life-Years, 10-Year Model Timeframe } \\
\hline \multirow[t]{19}{*}{0.20} & 0.24 & 0.28 & 0.32 & 0.36 & 0.40 & 0.44 & Parameter & $\begin{array}{l}\text { Low } \\
\text { Input }\end{array}$ & $\begin{array}{l}\text { High } \\
\text { Input }\end{array}$ & $\begin{array}{l}\text { Low } \\
\text { Result }\end{array}$ & $\begin{array}{l}\text { High } \\
\text { Result }\end{array}$ & Spread \\
\hline & & & & & & & Pediatric RR CR & 0.97 & 1.09 & 0.21 & 0.43 & 0.22 \\
\hline & & & & & & & Pediatric HR PFS & 0.41 & 0.61 & 0.38 & 0.25 & 0.13 \\
\hline & & & & & & & Pediatric RR Induction Death & 0.15 & 15.22 & 0.32 & 0.28 & 0.04 \\
\hline & & & & & & & Hyper-CVAD pr CR & 0.92 & 0.99 & 0.31 & 0.33 & 0.02 \\
\hline & & & & & & & $\%$ Transplant Candidates, Pediatric & 0.13 & 0.19 & 0.32 & 0.31 & 0.01 \\
\hline & & & & & & & $\%$ Transplant Candidates, Hyper-CVAD & 0.33 & 0.50 & 0.32 & 0.31 & 0.01 \\
\hline & & & & & & & Month Progression Risk Ends & 48 & 72 & 0.31 & 0.32 & 0.01 \\
\hline & & & & & & & pr 2nd CR & 0.00 & 0.26 & 0.32 & 0.32 & 0.00 \\
\hline & & & & & & & Hyper-CVAD pr Induction Death & 0.00 & 0.04 & 0.32 & 0.32 & 0.00 \\
\hline & & & & & & & pr Progression Survival & 0.86 & 0.99 & 0.32 & 0.32 & 0.00 \\
\hline & & & & & & & pr 2nd Progression & 0.83 & 0.97 & 0.32 & 0.32 & 0.00 \\
\hline & & & & & & & Hyper-CVAD pr CR After 1 Course & 0.75 & 0.87 & 0.32 & 0.32 & 0.00 \\
\hline & & & & & & & Hyper-CVAD 5-yr PFS & 0.36 & 0.52 & 0.32 & 0.32 & 0.00 \\
\hline & & & & & & & Utility: PFS, Induction & 0.52 & 0.78 & 0.32 & 0.32 & 0.00 \\
\hline & & & & & & & Utility: PFS, Intensification & 0.15 & 0.72 & 0.32 & 0.32 & 0.00 \\
\hline & & & & & & & Utility: PFS, Maintenance & 0.60 & 0.90 & 0.32 & 0.32 & 0.00 \\
\hline & & & & & & & Utility: PFS, Post-Therapy & 0.68 & 1.00 & 0.32 & 0.32 & 0.00 \\
\hline & & & & & & & Utility: Progression & 0.48 & 0.72 & 0.32 & 0.32 & 0.00 \\
\hline
\end{tabular}

FIG. 3. (A, B) Results of one-way sensitivity analysis. The impact of individual parameter ranges on model results is presented as tornado diagrams. Parameters with larger horizontal bars exhibit greater impacts on results. Gray bars indicate the low estimates in the parameter range and black bars indicate the high estimates in the parameter ranges. CR, complete response; HR, hazard ratio; QALYs, quality-adjusted life-years; pediatric, pediatric-inspired protocol; PFS, progression-free survival; pr, probability; RR, relative risk.

greater QALYs and life-years in at least $95 \%$ of simulations at both 5 and 10 years postinduction.

\section{Discussion}

To our knowledge, this exploratory analysis is the first comparative quantitative assessment of the potential benefits and harms associated with a pediatric-inspired protocol versus a hyper-CVAD protocol for the treatment of Philadelphia-negative AYA ALL. Results from the decision- analytic model suggest that AYA patients treated with the pediatric-inspired protocol experience a decrease in QALYs despite an increase in life-years versus hyper-CVAD during the initial stages of treatment. This decrease, although quite small, is consistent with the reported lower tolerability of pediatric protocols when they are used in AYAs, relative to use in a pediatric population. ${ }^{16,17}$ However, over the longer term, the lower quality of life associated with the induction and intensification phases of the pediatric-inspired treatment was largely offset by more favorable progression-free 
survival and overall survival in comparison with hyperCVAD. Notably, the pediatric-inspired protocol resulted in a slight increase in life-years throughout all treatment stages, including the induction phase.

These results support recent calls from a small contingent of stakeholders for utilizing pediatric treatment protocols, including asparaginase, in this patient population. ${ }^{22,23}$ Recent prospective clinical studies, as well as a meta-analysis, have also shown evidence of tolerability and survival that supports a pediatric-inspired treatment regimen in the AYA patient population. ${ }^{15,24-28}$ These studies have suggested encouraging survival outcomes for pediatric regimen-treated AYAs, with survival outcomes approaching those of younger children. ${ }^{13,29-32}$ The GRAALL 2003 study investigators retrospectively compared 214 pediatric protocol-treated AYA and adult patients (aged 15-60 years) with Philadelphia chromosome-negative ALL to 712 adult regimen-treated AYA and adult patients (aged 15-55 years) with Philadelphia chromosome-negative ALL in the LALA-94 trial. The pediatric-treated patients showed improvement in both 42month event-free survival (57\% vs. $33 \%$, respectively) and overall survival ( $61 \%$ vs. $41 \%$, respectively). ${ }^{23,28}$ Similar results were observed for event-free survival and overall survival in studies that have included both B-cell and T-cell ALL. ${ }^{29}$

More recently, Rytting et al. showed that 106 patients treated with augmented Berlin-Frankfurt-Münster therapy, including asparaginase, in AYAs were not associated with significant improvements in complete remission and overall survival compared with 102 patients receiving a hyperCVAD regimen. ${ }^{33}$ However, hyper-CVAD patients in this study with B-lymphocyte antigen CD20 expression $>20 \%$ received an augmented hyper-CVAD regimen with $\mathrm{CD} 20$ monoclonal antibodies, rituximab (45 patients) or ofatumumab (6 patients), which likely explains the similar outcomes between cohorts in this single-center study. We excluded this study from consideration in our analysis to focus on the comparison to a traditional hyper-CVAD regimen, but we recognize that augmented hyper-CVAD regimens represent a promising approach to treatment in populations with high CD20-positive expression.

The National Comprehensive Cancer Network (NCCN) recently listed pediatric-inspired protocols as the preferred regimen for AYA patients aged 15-39 years. ${ }^{34}$ However, despite the accumulating evidence suggesting the utility and benefits of pediatric protocols for the treatment of AYA patients with ALL, substantial barriers remain for the use of these protocols, including the lack of high-level evidence in the form of randomized controlled trials. In addition, AYAs represent a small group of patients, and this population is often treated by adult oncologists at a diversity of cancer centers and who therefore see fewer of these patients. ${ }^{16}$ While adverse events and intolerability have been considered a barrier to pediatric protocols, with certain toxicities (hepatotoxicity, in particular) reported as a more common problem in older individuals, accumulative evidence suggests the manageability of these toxicities. ${ }^{5}$

Of note, the Prospective U.S. Intergroup Trial C10403 demonstrated that pediatric-regimen toxicities were manageable, with low treatment-related mortality $(2 \%)$ and treatment with the pediatric regimen is feasible when administered by adult hematologist/oncologists to an AYA population aged up to 40 years. $^{35}$ A large $(N=1368)$ compassionate-use trial evaluated the safety and toxicity of asparaginase Erwinia chrysanthemi in patients of various ages. The study included 147 AYA patients (aged 16-39 years) and reported that the safety profile of asparaginase E. chrysanthemi in these patients was consistent with that found in the overall population. ${ }^{25}$

\section{Limitations}

Because there are no randomized controlled trials comparing the two treatment approaches being analyzed, the model relies on an indirect comparison between two studies from the literature. We chose these two studies after systematic literature review showed them to be the most comparable in regard to patient characteristics. However, they were not the same in all respects, and we had to rely on certain assumptions and adjustments. Furthermore, the model incorporated data from a specifically modified pediatric protocol, and thus the reported results may not necessarily be generalizable to other pediatric protocols. Similarly, the patient populations for both protocols were derived from studies that were not exclusively AYA patients, although data were extracted based on agestratified results within these studies. Additional limitations include the need for assumptions regarding outcomes in Philadelphia-negative patients, since the hyper-CVAD source included a mixed population with regard to this cytogenetic marker and assumptions for utilities due to lack of definitive estimates. Last, we excluded studies that included patients treated with an augmented hyper-CVAD regimen, which may bias our results to favor pediatric therapy.

\section{Conclusions}

Results from this decision-analytic model suggest that pediatric-inspired protocols may increase life-years throughout treatment stages and QALYs in the long term for AYA patients with Philadelphia-negative ALL. The model estimates that, while these patients may experience a decrease in QALYs versus hyper-CVAD during the initial stages of treatment for ALL, the pediatric-inspired protocol would lead to an overall increase both in life-years and QALYs in the longer term following the initial stages of treatment.

\section{Acknowledgments}

This study was funded by Jazz Pharmaceuticals, Inc. Under the direction of the authors, E. Jay Bienen, $\mathrm{PhD}$, and Cory Hussar, $\mathrm{PhD}$, employees of The Curry Rockefeller Group (CRG; Tarrytown, NY) provided medical writing assistance for this publication. Editorial assistance in formatting, proofreading, copy editing, and fact checking was also provided by CRG. Jazz Pharmaceuticals, Inc, provided funding to CRG for support in writing and editing this article.

\section{Author Disclosure Statement}

This analysis was supported by Jazz Pharmaceuticals, Inc. K.F.V., G.F.V., and V.L.F. are employees of Jazz Pharmaceuticals, Inc., who in the course of this employment have received stock options exercisable for, and other stock awards of, ordinary shares of Jazz Pharmaceuticals PLC. 
G.F.G. and D.L.V. were paid by Jazz Pharmaceuticals, Inc., as consultants for analyses in this study.

\section{References}

1. Mohan SR, Advani AS. Treatment of acute lymphoblastic leukemia in adolescents and young adults. J Adolesc Young Adult Oncol. 2011;1(1):19-24.

2. Howlader N, Noone A, Krapcho M, et al. SEER Cancer Statistics Review, 1975-2010, based on November 2012 SEER data submission. Bethesda, MD: National Cancer Institute; 2013. Accessed August 25, 2015 from: http://seer .cancer.gov/archive/csr/1975_2010/browse_csr.php?section SEL $=13$ \&pageSEL $=$ sect_13_table.12.html

3. Faderl S, Jeha S, Kantarjian HM. The biology and therapy of adult acute lymphoblastic leukemia. Cancer. 2003;98(7): 1337-54.

4. Nachman J. Clinical characteristics, biologic features and outcome for young adult patients with acute lymphoblastic leukaemia. Br J Haematol. 2005;130(2):166-73.

5. Stock W, Douer D, DeAngelo DJ, et al. Prevention and management of asparaginase/pegasparaginase-associated toxicities in adults and older adolescents: recommendations of an expert panel. Leuk Lymphoma. 2011;52(12): 2237-53.

6. Faderl S, Kantarjian HM, Talpaz M, Estrov Z. Clinical significance of cytogenetic abnormalities in adult acute lymphoblastic leukemia. Blood. 1998;91(11):3995-4019.

7. Moorman AV, Harrison CJ, Buck GA, et al. Karyotype is an independent prognostic factor in adult acute lymphoblastic leukemia (ALL): analysis of cytogenetic data from patients treated on the Medical Research Council (MRC) UKALLXII/Eastern Cooperative Oncology Group (ECOG) 2993 trial. Blood. 2007;109(8):3189-97.

8. Schlieben S, Borkhardt A, Reinisch I, et al. Incidence and clinical outcome of children with BCR/ABL-positive acute lymphoblastic leukemia (ALL). A prospective RT-PCR study based on 673 patients enrolled in the German pediatric multicenter therapy trials ALL-BFM-90 and CoALL05-92. Leukemia. 1996;10(6):957-63.

9. Crist W, Carroll A, Shuster J, et al. Philadelphia chromosome positive childhood acute lymphoblastic leukemia: clinical and cytogenetic characteristics and treatment outcome. A Pediatric Oncology Group study. Blood. 1990; 76(3):489-94.

10. Potosky AL, Harlan LC, Albritton K, et al. Use of appropriate initial treatment among adolescents and young adults with cancer. J Natl Cancer Inst. 2014;106(11):dju300.

11. Kantarjian HM, O'Brien S, Smith TL, et al. Results of treatment with hyper-CVAD, a dose-intensive regimen, in adult acute lymphocytic leukemia. J Clin Oncol. 2000; 18(3):547-61.

12. Garcia-Manero G, Kantarjian HM. The hyper-CVAD regimen in adult acute lymphocytic leukemia. Hematol Oncol Clin North Am. 2000;14(6):1381-96, x-xi.

13. Stock W, La M, Sanford B, et al. What determines the outcomes for adolescents and young adults with acute lymphoblastic leukemia treated on cooperative group protocols? A comparison of Children's Cancer Group and Cancer and Leukemia Group B studies. Blood. 2008; 112(5):1646-54.

14. Pui CH, Pei D, Campana D, et al. Improved prognosis for older adolescents with acute lymphoblastic leukemia. J Clin Oncol. 2011;29(4):386-91.
15. Ram R, Wolach O, Vidal L, et al. Adolescents and young adults with acute lymphoblastic leukemia have a better outcome when treated with pediatric-inspired regimens: systematic review and meta-analysis. Am J Hematol. 2012;87(5):472-8.

16. Schafer ES, Hunger SP. Optimal therapy for acute lymphoblastic leukemia in adolescents and young adults. Nat Rev Clin Oncol. 2011;8(7):417-24.

17. Breitenbach K, Stock W. Intergroup Trial C10403: a pediatric treatment approach to improve outcomes in adolescents and young adults with acute lymphoblastic leukemia. J Adolesc Young Adult Oncol. 2011;1(2):107-8.

18. Burke ME, Albritton K, Marina N. Challenges in the recruitment of adolescents and young adults to cancer clinical trials. Cancer. 2007;110(11):2385-93.

19. Kantarjian H, Thomas D, O'Brien S, et al. Long-term follow-up results of hyperfractionated cyclophosphamide, vincristine, doxorubicin, and dexamethasone (Hyper-CVAD), a dose-intensive regimen, in adult acute lymphocytic leukemia. Cancer. 2004;101(12):2788-801.

20. Storring JM, Minden MD, Kao S, et al. Treatment of adults with BCR-ABL negative acute lymphoblastic leukaemia with a modified paediatric regimen. Br J Haematol. 2009; 146(1):76-85.

21. Garrison LP, Jr., Towse A, Bresnahan BW. Assessing a structured, quantitative health outcomes approach to drug risk-benefit analysis. Health Aff (Millwood). 2007;26(3): 684-95.

22. Boissel N, Sender LS. Best practices in adolescent and young adult patients with acute lymphoblastic leukemia: a focus on asparaginase. J Adolesc Young Adult Oncol. 2015;4(3):118-28.

23. Dombret H, Cluzeau T, Huguet F, Boissel N. Pediatric-like therapy for adults with ALL. Curr Hematol Malig Rep. 2014;9(2):158-64.

24. Advani A, Sanford B, Luger S, et al. Frontline-treatment of acute lymphoblastic leukemia (ALL) in older adolescents and young adults (AYA) using a pediatric regimen is feasible: toxicity results of the prospective US Intergroup Trial C10403 (Alliance) [abstract]. Blood. 2013;122(21): Abstract 3903.

25. Plourde PV, Jeha S, Hijiya N, et al. Safety profile of asparaginase Erwinia chrysanthemi in a large compassionateuse trial. Pediatr Blood Cancer. 2014;61(7):1232-8.

26. Hayakawa F, Sakura T, Yujiri T, et al. Markedly improved outcomes and acceptable toxicity in adolescents and young adults with acute lymphoblastic leukemia following treatment with a pediatric protocol: a phase II study by the Japan Adult Leukemia Study Group. Blood Cancer J. 2014;4:e252.

27. Ibrahim A, Ali A, Mohammed MM. Outcome of adolescents with acute lymphoblastic leukemia treated by pediatrics versus adults protocols. Adv Hematol. 2014;2014:697675.

28. Huguet F, Leguay T, Raffoux E, et al. Pediatric-inspired therapy in adults with Philadelphia chromosome-negative acute lymphoblastic leukemia: the GRAALL-2003 study. J Clin Oncol. 2009;27(6):911-8.

29. Boissel N, Auclerc MF, Lheritier V, et al. Should adolescents with acute lymphoblastic leukemia be treated as old children or young adults? Comparison of the French FRALLE-93 and LALA-94 trials. J Clin Oncol. 2003; 21(5):774-80.

30. de Bont JM, Holt B, Dekker AW, et al. Significant difference in outcome for adolescents with acute lymphoblastic 
leukemia treated on pediatric vs adult protocols in the Netherlands. Leukemia. 2004;18(12):2032-5.

31. Hallbook H, Gustafsson G, Smedmyr B, et al. Treatment outcome in young adults and children $>10$ years of age with acute lymphoblastic leukemia in Sweden: a comparison between a pediatric protocol and an adult protocol. Cancer. 2006;107(7):1551-61.

32. Ramanujachar R, Richards S, Hann I, et al. Adolescents with acute lymphoblastic leukaemia: outcome on UK national paediatric (ALL97) and adult (UKALLXII/E2993) trials. Pediatr Blood Cancer. 2007;48(3):254-61.

33. Rytting ME, Jabbour EJ, Jorgensen JL, et al. Final results of a single institution experience with a pediatric-based regimen, the augmented Berlin-Frankfurt-Münster, in adolescents and young adults with acute lymphoblastic leukemia, and comparison to the hyper-CVAD regimen. Am J Hematol. 2016;91(8):819-23.

34. National Comprehensive Cancer Network, Inc. NCCN Clinical Practice Guidelines in Oncology: Acute Lympho- blastic Leukemia. Version 1.2016. Accessed September 12, 2016 from: www.nccn.org/patients/guidelines/all/\#

35. Stock W, Luger SM, Advani AS, et al. Favorable outcomes for older adolescents and young adults (AYA) with acute lymphoblastic leukemia (ALL): early results of US Intergroup Trial C10403. Blood. 2014;124(21):Abstract 796.

Address correspondence to: David L. Veenstra, PharmD, PhD Pharmaceutical Outcomes Research and Policy Program Department of Pharmacy University of Washington

H375 HSB

Box 357630

1959 NE Pacific Street Seattle, WA 98195-7630

E-mail: veenstra@uw.edu 